Southern Illinois University Edwardsville SPARK

SIUE Faculty Research, Scholarship, and Creative Activity

2018

\title{
Avian Habitat Use in a Chronosequence of Bottomland Hardwood Forest Restoration Sites
}

Paul T. Le

University of Colorado Denver, paul.le@ucdenver.edu

Lindley B. Ballen

Umpqua Community College

Richard Essner

ressner@siue.edu

Peter R. Minchin

Southern Illinois University Edwardsville, pminchi@siue.edu

Follow this and additional works at: https://spark.siue.edu/siue_fac

Part of the Biology Commons, and the Ecology and Evolutionary Biology Commons

\section{Recommended Citation}

Le, Paul T.; Ballen, Lindley B.; Essner, Richard; and Minchin, Peter R., "Avian Habitat Use in a Chronosequence of Bottomland Hardwood Forest Restoration Sites" (2018). SIUE Faculty Research, Scholarship, and Creative Activity. 89.

https://spark.siue.edu/siue_fac/89 


\title{
Avian Habitat Use in a Chronosequence of Bottomland Hardwood Forest-Restoration Sites
}

\author{
Paul T. Le ${ }^{1,2, *}$, Lindley B. Ballen ${ }^{1,3}$, Richard L. Essner ${ }^{1}$, and Peter R. Minchin ${ }^{1}$
}

\begin{abstract}
Since the 1950s, anthropogenic activity has caused the loss of millions of hectares of bottomland hardwood forest in the Upper Mississippi River Valley, causing population declines in bird populations. Restoration of these forest stands has been ongoing for the past 2 decades. We assessed bird species presence on sites in the Upper Mississippi River Valley to quantify diversity and relate presence to habitat conditions and sites' age since restoration. We observed higher mean diversities at mature bottomland-forest sites during the spring and autumn, but nested ANOVAs indicated no significant differences among restoration-age categories during spring. During the autumn, the 15-23-y and the mature bottomland-forest categories were significantly different from the $<7$-y category. Predictive habitat models differed among species, but presence of forest-dwelling birds was positively related to forested conditions, such as tree height and tree density. Overall, our analyses show that a variety of birds use these sites, and we suggest further exploration of how assemblages may change in future surveys.
\end{abstract}

\section{Introduction}

The Upper Mississippi River Valley (UMR) represents a dynamic region in which species diversity and ecosystem function rely heavily on regular flood pulses (Knutson et al. 1996, Romano 2010, Sparks 2010). Many resident and migratory species in this area depend on these annual flood regimes to provide ecosystem services, such as food, habitat, recruitment, and connectivity (Garvey et al. 2010, Romano 2010, Twedt and Loesch 1999). Historically, bottomland hardwood forests dominated the landcover and were represented by millions of hectares of contiguous floodplains. However, there has been significant forest-habitat loss in the UMR attributed to: (1) the development of locks and dams for commercial navigation, which altered the flood regime; and (2) the conversion of bottomland hardwood forests to agriculture and (sub)urbanized areas, which fragmented the historically contiguous landscape (Kirsch et al. 2013, Twedt and Loesch 1999). This fragmentation inhibited vital ecosystem services such as water enhancement and nutrient cycling (King and Keeland 1999). Additionally, historical spring floods typically receded by mid-May, but the systems of locks and dams increased the intensity of flooding in many areas (Knutson and Klaas 1997).

\footnotetext{
${ }^{1}$ Department of Biological Sciences, Southern Illinois University Edwardsville, Campus Box 1651, Edwardsville, IL 62026. ${ }^{2}$ Department of Integrative Biology, University of Colorado Denver, Campus Box 171, PO Box 173364, Denver, CO 80217. ${ }^{3}$ Umpqua Community College, 1140 Umpqua College Rd, Roseburg, OR 97471. * Corresponding author - paul.le@ucdenver.edu.
}

Manuscript Editor: Jeremy Kirchman 
Surveys within the UMR found that floodplain forests provide habitat for $\sim 290$ species of birds (Nelson and Wlosinski 1999). Millions of birds annually use these habitats for breeding, migrating, and overwintering. Presently, trends from 1966-2015 show that $>33 \%$ of UMR birds have experienced population declines due to habitat loss and fragmentation in bottomland forests in the UMR (King et al. 2006, Robinson et al. 1995, Sauer et al. 2017). Some species of conservation concern, such as Coccyzus erythropthalmus (Wilson) (Black-billed Cuckoo) and Setophaga cerulea (Wilson) (Cerulean Warbler) preferentially use the UMR as a migratory corridor (Kirsch et al. 2013, Knutson et al. 1999, Thompson et al. 2012). As such, some land areas, such as Two Rivers National Wildlife Refuge and Riverlands Migratory Bird Sanctuary, have been designated as Globally Important Bird Areas to facilitate protection of bird species (Jensen 2007, Knutson et al. 1999, Wells et al. 2005).

To create more suitable habitat for species that utilize the UMR, over the past 2 decades, the US Army Corps of Engineers (USACE) has aided in the restoration of bottomland hardwood forests in the area, which includes $\sim 1.2$ million ha ( $\sim 3$ million ac) of floodplain forests (Sparks 2010, Theiling et al. 2015, Twedt and Portwood 1997). Historically, bottomland hardwood forests were primarily a mixture of Quercus (oak), Ulmus (elm), and Acer (maple), which are late-successional species (Hanberry et al. 2012, Romano 2006). Therefore, restorations have included planting of root-production method (RPM) seedlings with highly developed root systems, which increase their survival and initial vertical growth (Dey et al. 2004).

Restoration efforts in the lower Mississippi River Valley have been well described (Hamel 2003; Twedt et al. 2002, 2008). However, restoration sites in the UMR have not received as much research attention, and little is known regarding species composition of the avian assemblages that utilize these areas. This information is critical for informing conservation and management strategies for bird species that have historically used the UMR. We offer one of the first exploratory studies to survey restoration sites in the UMR, the results of which provide new information useful to the conservation and management mission of wildlife refuges and the USACE along the Mississippi Flyway. Our objectives were to (1) quantify species diversity of avian assemblages that were present during spring and autumn, and (2) model bird species presence as a function of habitat conditions. We formulated 2 hypotheses based on our objectives. (1) We expected species diversity to peak in sites that were 15-23 years since restoration. Previous studies have shown that there is higher bird diversity at open woodland sites when compared to grassland and forest sites (Au et al. 2008, Brawn 2006, Davis et al. 2000). This hypothesis also corresponds to the intermediate disturbance hypothesis, which states that there is likely to be greater diversity due to more habitat heterogeneity in habitats with regular disturbances (Connell 1978, Roxburgh et al. 2004). (2) We expected habitat models to generally show that birds associate with vegetation variables present at the sites where they are traditionally present. For example, that there would be a higher likelihood of detecting a Cerulean Warbler at mature bottomland forest sites than at younger, open-woodland sites (Kirsch et al. 2013). 


\section{Field Site Description}

We surveyed 9 bottomland hardwood-forest restoration sites (1-23 years since restoration) in Illinois and Missouri, located near either the Illinois or Mississippi Rivers (Fig. 1). Four sites (Epping, Chain of Rocks, Earth Day Patch, and American Bottoms) are within the St. Louis metropolitan area and border suburban communities. We selected only sites that were $\geq 3$ ha and planted with RPM seedlings of native oak species. In addition, we surveyed 2 mature ( $>70 \mathrm{y}$ old) bottomland hardwood-forest sites (American Bottoms and Rip Rap Landing) as references. We collected avian and vegetation data from 5 plots at each site. We used ArcGIS Desktop 10 (ESRI 2010) to randomly select plots with the constraint that points were at least 50-m apart to reduce sampling overlap and, to limit edge effects, could not be within a 30-m buffer zone. All plots were at least $150 \mathrm{~m}$ apart in our final plot selection. The Rip Rap Landing site plots were derived from preestablished plots from an earlier study. Following plot selection, we used a GPS receiver (Garmin GPSMAP 62S, Model 010-00868-01, Olathe, KS) to record the locations of sample plots. We marked each plot with a $1.83-\mathrm{m}$ steel t-post.

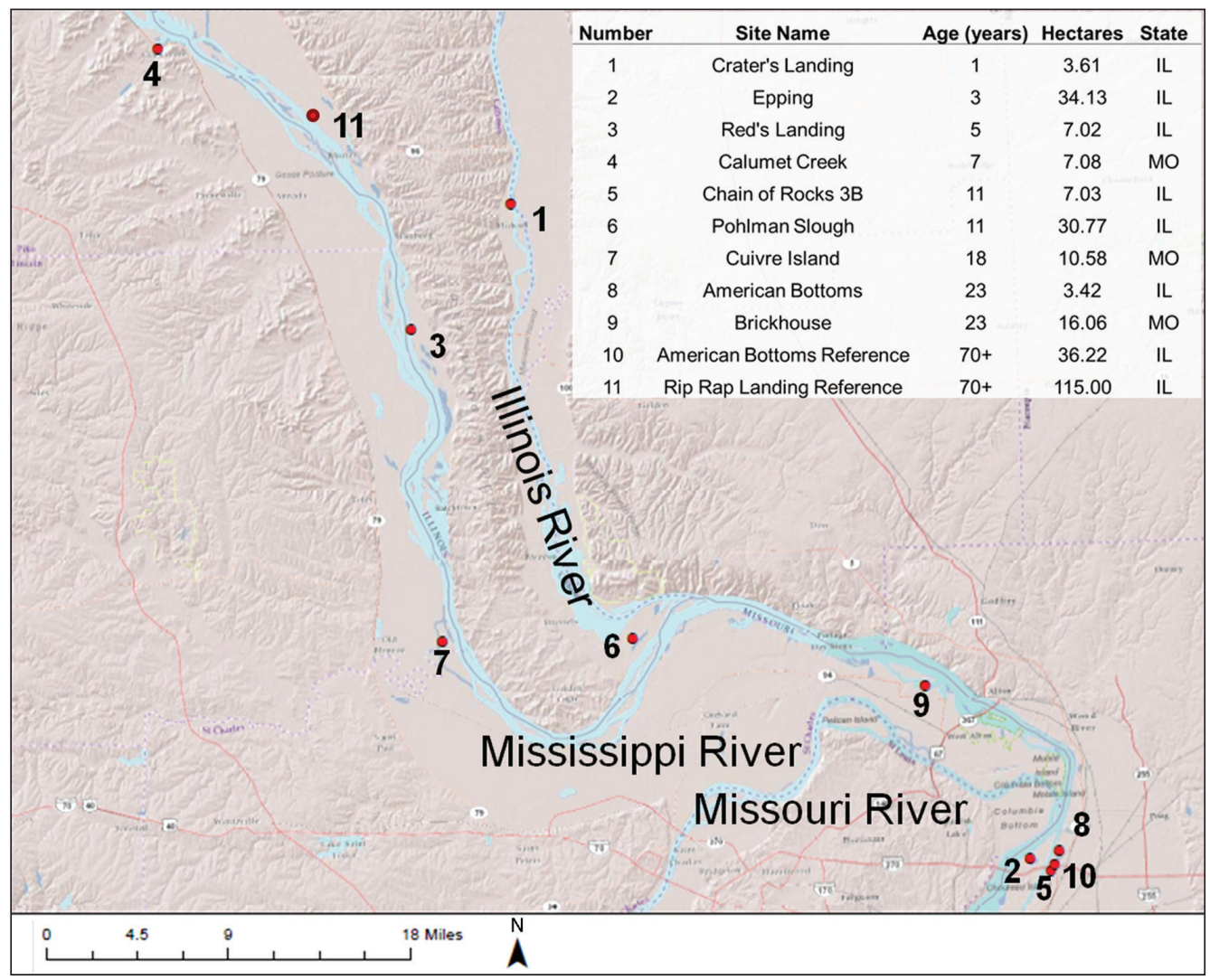

Figure 1. We chose 9 restoration sites and 2 mature bottomland-forest reference sites for data collection. All sites border along either the Illinois or Mississippi Rivers. Rip Rap Landing covers a large area and includes varying ages of succession. The USACE estimated that $\sim 115$ ha at that site is mature bottomland hardwood forest. 


\section{Methods}

\section{Avian point-count surveys}

We used 25-m fixed-radius point-count surveys to survey birds during spring and autumn over 3 sampling periods: 1 May-30 June 2013 (spring), 1 September-31 October 2013 (autumn), and 10 May-30 June 2014 (spring) (Hutto et al. 1986). Due to excessive flooding during spring 2013 and 2014, we could access only 8 of the 11 sites for spring surveys. We visited all plots 3 times during each season to account for variation in arrival timing for different species and because we wanted to survey a variety of species. We conducted bird surveys between $0600 \mathrm{~h}$ and $1000 \mathrm{~h}$ for a 10 -min duration after a 5-min acclimation period. We determined the 25-m boundary using a laser rangefinder (Nikon ProStaff 550, Model 8369, Melville, NY), to prevent sampling outside of the fixed radius (Richter et al. 2010). We avoided surveying birds during inclement weather, such as fog, rain, or high winds.

\section{Vegetation surveys}

We surveyed non-woody vegetation 15 May-1 September 2013 and woody vegetation 15 September-15 November 2013 and in early March 2014 during a single sample visit (Table 1 ). We created $17.84-\mathrm{m}$-radius vegetation plots within the 25-m-radius bird-survey plots, (Fig. 2). In the vegetation plots, we recorded tree species, crown density, diameter at breast height (DBH), and abundance for trees that were at least $5 \mathrm{~cm}$ in diameter at a height of $1.4 \mathrm{~m}$ up from the base of the trunk. We calculated tree dominance by totaling the cross-sectional area of all trees and estimating the total area in which trees occurred per hectare.

Within vegetation plots, we surveyed the shrub and ground vegetation of 4 belts in which we recorded the number of shrub stems that were $\geq 1 \mathrm{~m}$ in height (Fig. 2). We recorded forb and grass data, vegetation height, and litter depth in five $0.5-\mathrm{m}^{2}$ quadrats along the belt. We placed the first quadrat $1 \mathrm{~m}$ away from the center and

Table 1. We included 12 geographic and vegetation variables in predictive habitat models. We chose them based on parameters that were presumed to be important to a species' presence or absence. ${ }^{*}$ denotes variables with curvilinear relationships with bird species presence, the values of which were squared in the models.

\begin{tabular}{|c|c|c|}
\hline Variable & Description of variable & Measurement format \\
\hline Landsize & Area of site & Continuous $\left(\mathrm{m}^{2}\right)$ \\
\hline DistEdge $^{*}$ & Distance to edge & Continuous (m) \\
\hline DistRiver & Distance to nearest river or canal & Continuous (m) \\
\hline GroundCover $^{*}$ & Ground layer cover & Categorical (midpoint percentage) \\
\hline DBH & Diameter at breast height $\left(90^{\text {th }}\right.$ percentile $)$ & Continuous $(\mathrm{cm})$ \\
\hline TreeHeight & Height of tree $\left(90^{\text {th }}\right.$ percentile $)$ & Continuous (m) \\
\hline LitterDepth & Litter depth & Continuous $(\mathrm{cm})$ \\
\hline HerbHeight $^{*}$ & Average maximum grass/forb height & Continuous (m) \\
\hline ShrubHeight ${ }^{*}$ & Average maximum shrub height & Continuous (m) \\
\hline CrownDensity & Average tree-crown density & Categorical (1 [lowest] -9 [highest $])$ \\
\hline TreeDom & Tree dominance & Continuous $\left(\mathrm{m}^{2} / \mathrm{ha}\right)$ \\
\hline ShrubDensity & Shrub density & Continuous (stems/ha) \\
\hline
\end{tabular}


spaced subsequent quadrats 3-m apart (Fig. 2). We used general percent-cover estimates for the quadrats using Braun-Blanquet classes (Van Der Maarel 1975).

\section{Data analyses}

Following the guidelines set in a previous study in bottomland-hardwood forests, we divided sites into 4 age categories based on vegetative characteristics: $<7$ years since restoration (grassland-like), 7-14 years since restoration (shrublandlike), 15-23 years since restoration (open woodland-like), and mature bottomlandforest reference sites (forest-like) (Wilson and Twedt 2005). We utilized these categories to identify trends in diversity and apparent frequencies of occurrence for bird species through nested ANOVA measures.

For each plot, we calculated diversity with the following diversity indices:

$$
\begin{aligned}
& \text { antilog Shannon's }=e\left(\sum_{i=1}^{\mathrm{R}} p_{i} \ln p_{i}\right) \\
& \text { inverse Simpson's }=1 /\left(\sum_{i=1}^{\mathrm{R}} p_{i}{ }^{2}\right)
\end{aligned}
$$

We chose to calculate the antilog Shannon's and inverse Simpson's diversity indices to facilitate interpretation; if all species had the same number of individuals, diversity would equal richness (Möckel et al. 2016, Pizzio et al. 2016). We performed nested ANOVAs and Tukey-Kramer comparison tests on diversity data to determine differences in diversity as a function of restoration-age category in NCSS 2007 statistical software (Hintze 2007; NCSS, LLC, Kaysville, UT). Survey plots were nested within sites, which were nested within age categories.

Figure 2. Sample design of the avian and vegetation plots. The outermost circle represents the area in which avian point-count surveys were conducted. We included in statistical analyses all birds seen within the $25-\mathrm{m}$ radius. The inner dotted circle represents the area in which vegetation surveys occurred. We tagged and identified all trees within the $17.84-\mathrm{m}$ radius. We collected shrub data within the belts and forb and vegetation-cover data within each quadrat.

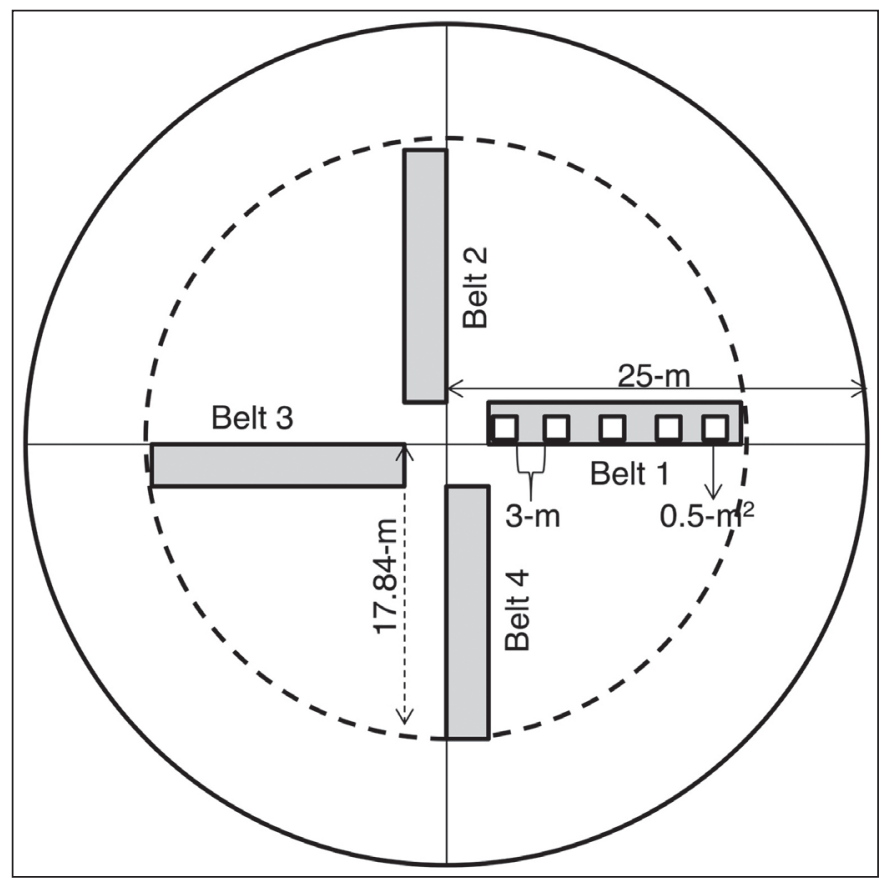


We used logistic regression to create predictive habitat-models, and interpreted the models' ability to effectively estimate selection probabilities (Keating and Cherry 2004). We generated separate models for spring and autumn survey periods. We chose logistic regression because we developed presence/absence models, which employ vegetation and landsize variables to estimate the probability of a species utilizing an area. We considered presence/absence modeling to be the most appropriate because many of the birds surveyed were short-term migrants occupying areas for only short periods, making it challenging to estimate abundances. We squared variables that had a curvilinear relationship with species presence in anticipation that influential variables might not be a predicted indicator due to a lack of a linear response (Austin 2007). We modeled birds that were present in at least 5 of 55 plots within our sites. We present birds with at least 1 significant indicator from their best model.

We did not propose or run a priori candidate models because of the number of species we surveyed and the lack of detailed descriptions for some bird species. We used SAS 9.3 (SAS Institute, Cary NC) to write a predictive-model logistic procedure to produce all possible models using a combination of all variables for each species in. All of our models reported Akaike information criterion (AIC) values, which represent the quality of a model based on a particular set of variables (Akaike 1974). We recorded the best model for each species, represented by the lowest AIC, and we standardized all associated variables in the best-fitting model by calculating $Z$-scores. We also recorded the apparent frequency of the bird species occurring on a specific plot from the model and performed nested ANOVAs and Tukey-Kramer post hoc tests to compare significant differences in apparent frequencies of occurrence in varying age categories in NCSS 2007 (Hintze 2007).

\section{Results}

\section{Species diversity}

During the spring 2013 and 2014 field seasons, we observed 65 bird species within 40 sample plots (Table 2). During the autumn 2013 field season, we observed 79 bird species within 55 sample plots (Table 2).

Although we observed higher mean diversities at mature bottomland-forest sites during the spring and autumn, nested ANOVAs indicated no significant differences among restoration-age categories during spring $\left(F_{3,4}=0.52, P=0.689\right.$ [antilog Shannon's], $F_{3,4}=0.65, P=0.625$ [Simpson's]; Fig. 3a, b). During the autumn, the $15-23-y$ and the mature bottomland-forest categories were significantly different from the $<7$-y category $\left(F_{3,6}=8.18, P=0.015\right.$ [antilog Shannon's], $F_{3,6}=7.96, P=$ 0.016 [Simpson's]; Fig. 3c, d).

\section{Habitat models}

Grassland birds. Spizella pusilla (Wilson) (Field Sparrow) presence during spring was negatively associated with tree height $(\beta=-1.34, P=0.029)$, whereas during autumn their presence was associated with distance to the edge of the site $(\beta=1.13, P=0.047)$ and $\mathrm{DBH}(\beta=-1.70, P=0.030)$ (Table 3$)$. 
Table 2. Bird species observed within the 25 - $\mathrm{m}$ fixed-radius plots during spring $(n=65)$ and autumn $(n=79) .{ }^{*}$ denotes species seen only in spring, ${ }^{* *}$ denotes species seen only in autumn. [Table continued on following page.]

Order Galliformes

Family Phasianidae: Bonasa umbellus (L.) (Ruffed Grouse) ${ }^{* *}$

Order Columbiformes

Family Columbidae: Columba livia Gmelin (Rock Pigeon) ${ }^{*}$, Zenaida macroura (L.) (Mourning Dove) ${ }^{* *}$

Order Cuculiformes

Family Cuculidae: Coccyzus americanus (L.) (Yellow-billed Cuckoo) ${ }^{* *}$

Order Apodiformes

Family Apodidae: Chaetura pelagica (L.) (Chimney Swift) ${ }^{* *}$

Family Trochilidae: Archilochus colubris (L.) (Ruby-throated Hummingbird)

Order Charadriiformes

Family Charadriidae: Charadrius vociferous L. (Killdeer) ${ }^{* *}$

Order Coraciiformes

Family Alcedinidae: Megaceryle alcyon (L.) (Belted Kingfisher) ${ }^{* *}$

Order Piciformes

Family Picidae: Melanerpes erythrocephalus (L.) (Red-headed Woodpecker), Melanerpes carolinus (L.) (Red-bellied Woodpecker), Sphyrapicus varius (L.) (Yellow-bellied Sapsucker) ${ }^{* *}$, Picoides pubescens (L.) (Downy Woodpecker), Picoides villosus (L.) (Hairy Woodpecker), Colaptes auratus (L.) (Northern Flicker), Dryocopus pileatus (L.) (Pileated Woodpecker) ${ }^{* *}$

Order Passeriformes

Family Tyrannidae: Contopus virens (L.) (Eastern Wood-Pewee), Empidonax virescens (Vieillot) (Acadian Flycatcher) $^{* *}$, Empidonax alnorum Brewster (Alder Flycatcher) ${ }^{* *}$, Sayornis phoebe (Latham) (Eastern Phoebe), Tyrannus tyrannus (L.) (Eastern Kingbird)

Family Laniidae: Lanius excubitor Campbell (Northern Shrike) ${ }^{* *}$

Family Vireonidae: Vireo bellii Audubon (Bell's Vireo) ${ }^{* *}$, Vireo gilvus Vieillot (Warbling Vireo) ${ }^{* *}$, Vireo olivaceus (L.) (Red-eyed Vireo)

Family Corvidae: Cyanocitta cristata (L.) (Blue Jay), Corvus brachyrhynchos Brehm (American Crow)

Family Hirundinidae: Tachycineta bicolor (Vieillot) (Tree Swallow), Riparia riparia (L.) (Bank Swallow)

Family Paridae: Poecile carolinensis (Audubon) (Carolina Chickadee), Baeolophus bicolor L. (Tufted Titmouse)

Family Sittidae: Sitta carolinensis Latham (White-breasted Nuthatch)

Family Certhiidae: Certhia americana Bonaparte (Brown Creeper) ${ }^{* *}$

Family Troglodytidae: Thryothorus ludovicianus (Latham) (Carolina Wren)

Family Polioptilidae: Polioptila caerulea (L.) (Blue-gray Gnatcatcher) ${ }^{* *}$

Family Turdidae: Catharus ustulatus (Tschudi) (Swainson's Thrush), Hylocichla mustelina (Gmelin) (Wood Thrush), Turdus migratorius L. (American Robin)

Family Mimidae: Dumetella carolinensis (L.) (Gray Catbird) ${ }^{* *}$, Toxostoma rufum (L.) (Brown Thrasher), Mimus polyglottos (L.) (Northern Mockingbird)

Family Fringillidae: Spinus tristis (L.) (American Goldfinch)

Family Passerellidae: Pipilo erythrophthalmus (L.) (Eastern Towhee), Spizella passerina (Bechstein) (Chipping Sparrow), Spizella pallida (Clay-colored Sparrow) ${ }^{* *}$, Spizella pusilla (Swainson) (Field Sparrow), Pooecetes gramineus (Gmelin) (Vesper Sparrow), Chondestes grammacus (Say) (Lark Sparrow), Passerculus sandwichensis (Gmelin) (Savannah Sparrow), Ammodramus savannarum (Gmelin) (Grasshopper Sparrow), Melospiza melodia (Wilson) (Song Sparrow), Melospiza lincolnii (Audubon) (Lincoln's Sparrow) ${ }^{* *}$, Junco hyemalis (L.) (Dark-eyed Junco) $^{* *}$

Family Icteriidae: Icteria virens (L.) (Yellow-breasted Chat)

Family Icteridae: Sturnella magna (L.) (Eastern Meadowlark), Icterus spurius (L.) (Orchard Oriole) ${ }^{*}$, Icterus galbula (L.) (Baltimore Oriole)*, Agelaius phoeniceus (L.) (Red-winged Blackbird), Molothrus ater (Boddaert) (Brown-headed Cowbird), Euphagus cyanocephalus (Wagler) (Brewer's Blackbird) 
Table 2, continued.

Family Parulidae: Seiurus aurocapilla (L.) (Ovenbird) ${ }^{* *}$, Helmitheros vermivorum (Gmelin) (Worm-eating Warbler) ${ }^{*}$, Parkesia noveboracensis (Gmelin) (Northern Waterthrush) ${ }^{* *}$, Vermivora cyanoptera (L.) (Blue-winged Warbler), Mniotilta varia (L.) (Black-and-white Warbler), Protonotaria citrea (Boddaert) (Prothonotary Warbler) ${ }^{*}$, Oreothlypis peregrina (Wilson) (Tennessee Warbler), Oreothlypis celata (Say) (Orange-crowned Warbler)*, Geothlypis formosa (Wilson) (Kentucky Warbler)*, Geothlypis trichas (L.) (Common Yellowthroat), Setophaga ruticilla (L.) (American Redstart), Setophaga cerulea (Wilson) (Cerulean Warbler), Setophaga americana (L.) (Northern Parula), Setophaga fusca (Muller) (Blackburnian Warbler), Setophaga striata (Forster) (Blackpoll Warbler), Setophaga caerulescens (Gmelin) (Black-throated Blue Warbler) ${ }^{*}$, Setophaga coronata (L.) (Yellow-rumped Warbler), Setophaga magnolia (Wilson) (Magnolia Warbler), Setophaga petechia (L.) (Yellow Warbler), Setophaga discolor (Vieillot) (Prairie Warbler)*, Setophaga dominica (L.) (Yellow-throated Warbler) $^{*}$, Cardellina canadensis (L.) (Canada Warbler) ${ }^{* *}$, Cardellina pusilla (Wilson) (Wilson's Warbler)

Family Cardinalidae: Piranga rubra* (L.) (Summer Tanager), Piranga olivacea (Gmelin) (Scarlet Tanager), Cardinalis cardinalis (L.) (Northern Cardinal), Pheucticus ludovicianus (L.) (Rosebreasted Grosbeak), Passerina cyanea (L.) (Indigo Bunting)*
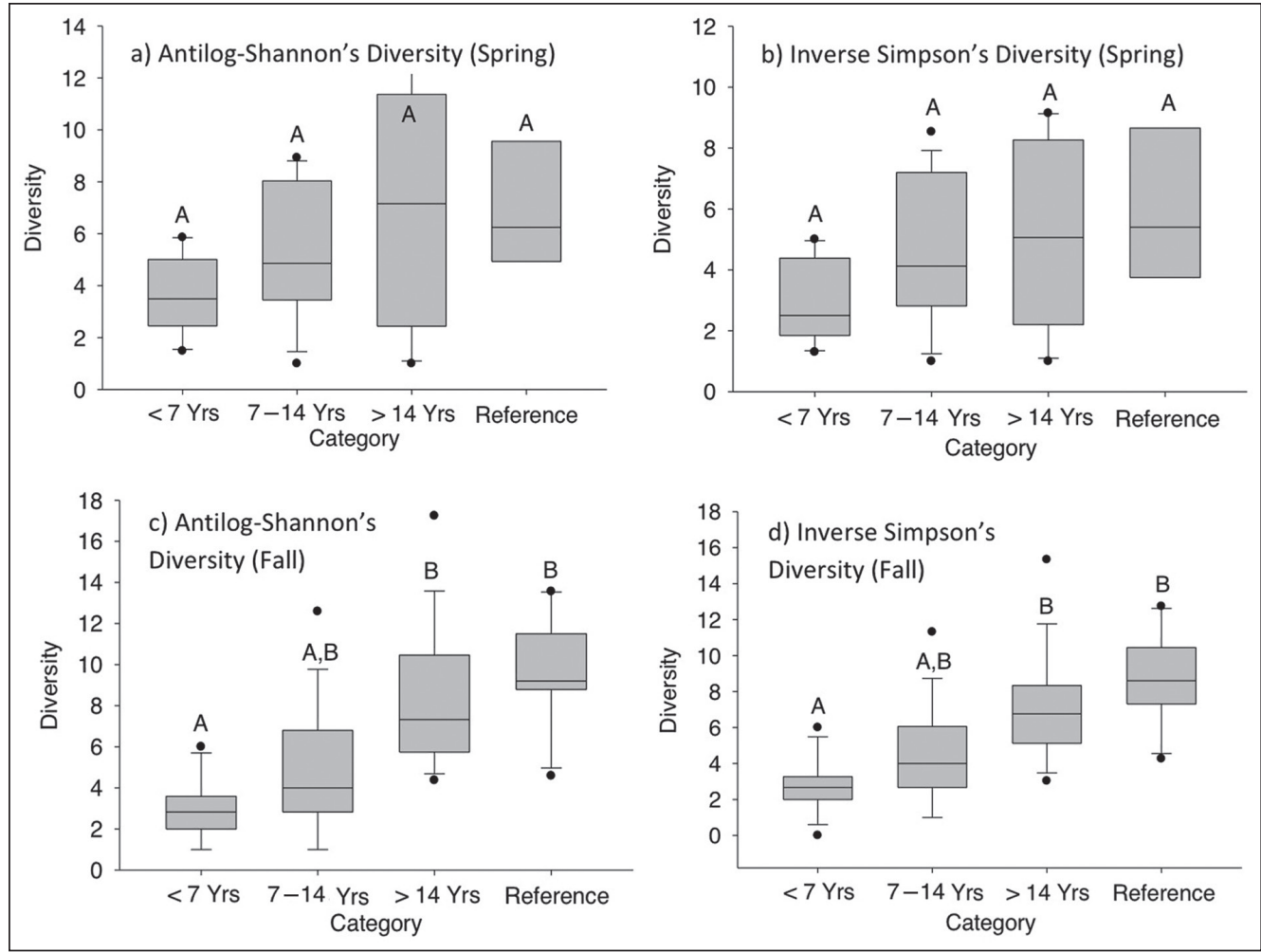

Figure 3. Diversity indices for each restoration category during spring and autumn surveys. Median (bar), $10^{\text {th }}$ (lower error bar), $25^{\text {th }}$ (lower box), $75^{\text {th }}$ (upper box), and $90^{\text {th }}$ (upper error bar) percentiles are included. Outliers are represented with black dots. Points with the same letter represent no significant difference between means, which is based on Tukey-Kramer post hoc tests at $P=0.05$. 
Table 3. The following are the best habitat models for species as determined by the lowest AIC value and regression analyses. Variables that are significant at $P=0.05$ are designated with $\left({ }^{*}\right)$. All variables have been standardized.

\begin{tabular}{|c|c|}
\hline Common Name & Model \\
\hline \\
\hline $\begin{array}{l}\text { Grassland Birds } \\
\text { Field Sparrow }\end{array}$ & TreeHeight $\left(-1.34^{*}\right)$ \\
\hline $\begin{array}{l}\text { Shrubland Birds } \\
\text { Common Yellowthroat }\end{array}$ & $\begin{array}{l}\text { GroundCover }\left(1.42^{*}\right) \text { TreeHeight (-2.16) ShrubHeight (-3.64) } \\
\text { ShrubDensity (1.51) ShrubHeight2 (1.89) }\end{array}$ \\
\hline Red-winged Blackbird & TreeDom $\left(-7.59^{*}\right)$ \\
\hline $\begin{array}{l}\text { Open Woodland Birds } \\
\text { American Goldfinch }\end{array}$ & $\begin{array}{l}\text { DistEdge }\left(4.62^{*}\right) \text { TreeDom }(-2.54) \text { DistEdge }{ }^{2}\left(-4.27^{*}\right) \\
\text { HerbHeight }^{2}(0.41)\end{array}$ \\
\hline $\begin{array}{l}\text { American Robin } \\
\text { Indigo Bunting }\end{array}$ & $\begin{array}{l}\text { CrownDensity }\left(0.96^{*}\right) \text { TreeDom }(1.41) \text { ShrubDensity }\left(0.81^{*}\right) \\
\text { DistRiver }\left(-0.94^{*}\right) \text { GroundCover }\left(0.60^{*}\right) \text { ShrubDensity }\left(-0.61^{*}\right) \\
\text { HerbHeight }^{2}\left(0.76^{*}\right)\end{array}$ \\
\hline Northern Cardinal & LitterDepth $\left(1.10^{*}\right)$ \\
\hline Summer Tanager & ShrubDensity $\left(0.46^{*}\right)$ \\
\hline Yellow Warbler & $\begin{array}{l}\text { DistRiver (-1.96) GroundCover }\left(1.62^{*}\right) \text { LitterDepth }(0.78) \\
\text { TreeDom (1.89) ShrubDensity }(1.42)\end{array}$ \\
\hline \multicolumn{2}{|l|}{ Forest Birds } \\
\hline Northern Parula & $\begin{array}{l}\text { DistRiver (-3.32) GroundCover (1.21) TreeDom }\left(3.12^{*}\right) \\
\text { ShrubDensity (1.10) HerbHeight2 (1.27) }\end{array}$ \\
\hline \multicolumn{2}{|l|}{ Autumn } \\
\hline Grassland Birds & DistEdge $\left(1.13^{*}\right) \mathrm{DBH}\left(-1.70^{*}\right)$ \\
\hline $\begin{array}{l}\text { Shrubland Birds } \\
\quad \text { Yellow-breasted Chat }\end{array}$ & DBH (0.30) ShrubDensity (-0.39) GroundCover $\left(0.77^{*}\right)$ \\
\hline $\begin{array}{l}\text { Open Woodland Birds } \\
\text { Eastern Phoebe } \\
\text { Gray Catbird } \\
\text { Hermit Thrush } \\
\text { Northern Cardinal } \\
\text { Northern Flicker } \\
\text { Red-headed Woodpecker }\end{array}$ & $\begin{array}{l}\text { DBH }\left(0.97^{*}\right) \text { TreeDom }(-0.71) \\
\text { Landsize }(-1.64) \text { DBH }\left(1.37^{*}\right) \text { ShrubDensity }(0.77) \\
\text { Landsize }(-2.42) \text { BH }\left(4.54^{*}\right) \text { TreeHeight }\left(-3.61^{*}\right) \text { ShrubHeight }{ }^{2}(-7.91) \\
\text { Landsize }\left(-3.52^{*}\right) \text { DBH }\left(6.52^{*}\right) \text { TreeHeight }\left(-2.41^{*}\right) \\
\text { GroundCover }(-0.48) \text { TreeDom }\left(0.48^{*}\right) \\
\text { DistRiver }(0.73) \text { GroundCover }(-1.87) \text { TreeHeight }\left(1.50^{*}\right) \\
\text { LitterDepth }(-1.24) \text { CrownDensity }(1.55) \text { TreeDom }(-0.98) \\
\text { HerbHeight }^{2}(0.94)\end{array}$ \\
\hline \multicolumn{2}{|l|}{ Forest Birds } \\
\hline Carolina Chickadee & $\begin{array}{l}\text { DBH }\left(1.78^{*}\right) \text { LitterDepth }(-0.77) \text { CrownDensity }(-0.29) \\
\text { DistEdge }^{2}\left(-1.72^{*}\right) \text { ShrubHeight }^{2}(0.39)\end{array}$ \\
\hline Downy Woodpecker & $\begin{array}{l}\text { TreeHeight }\left(0.71^{*}\right) \text { HerbHeight (0.47) ShrubDensity }(0.52) \\
\text { Ground } \operatorname{Cover}^{2}\left(-1.34^{*}\right)\end{array}$ \\
\hline Eastern Wood-Pewee & $\begin{array}{l}\text { DistRiver }\left(0.44^{*}\right) \text { GroundCover }\left(0.59^{*}\right) \text { TreeHeight }(0.35) \\
\text { ShrubHeight }\left(-2.48^{*}\right) \text { ShrubHeight2 }\left(2.47^{*}\right)\end{array}$ \\
\hline Northern Parula & TreeDom $\left(0.54^{*}\right)$ \\
\hline Red-eyed Vireo & DistRiver (-0.59) DBH (-0.69) TreeHeight $\left(1.12^{*}\right)$ HerbHeight $(0.36)$ \\
\hline White-breasted Nuthatch & Landsize $(-1.77)$ TreeHeight $\left(0.98^{*}\right)$ HerbHeight $^{2}(-0.69)$ \\
\hline White-throated Sparrow & $\begin{array}{l}\text { DistRiver }\left(0.65^{*}\right) \text { DBH }\left(4.04^{*}\right) \text { TreeHeight }(-1.85) \\
\text { CrownDensity }(-0.43) \text { TreeDom }\left(-2.46^{*}\right)\end{array}$ \\
\hline
\end{tabular}


Shrubland birds. We detected no particular trends for shrubland birds. During spring, Geothlypis trichas (L.) (Common Yellowthroat) presence was associated with groundcover $(\beta=1.42, P=0.039)$, and Agelaius phoeniceus (L.) (Red-winged Blackbird) presence was associated with tree dominance $(\beta=-7.59, P=0.044)$ (Table 3). During autumn, Icteria virens (L.) (Yellow-breasted Chat) had a positive association with the curvilinear relationship to groundcover $(\beta=0.77, P=0.026)$; Table 3).

Open woodland birds. During autumn, the presence of many open-woodland birds was positively associated with DBH (Table 3). Additionally, tree height differed and had significant negative associations with Catharus guttatus (Pallas) (Hermit Thrush) $(\beta=-3.61, P=0.043)$ and Cardinalis cardinalis (L.) (Northern Cardinal) during the autumn $(\beta=-2.41, P=0.036)$, but was positively associated with Melanerpes erythrocephalus (L.) (Red-headed Woodpecker) $(\beta=1.50, P=$ 0.044 (Table 3). Further, during spring, some open-woodland birds exhibited associations with shrub density (Table 3 ).

Forest birds. Several models for forest birds showed significant relationships with tree variables. Three of the autumn forest-bird models had positive associations with tree height: Picoides pubescens (L.) (Downy Woodpecker) $(\beta=0.71, P=$ $0.008)$, Vireo olivaceus (L.) (Red-eyed Vireo) $(\beta=1.12, P=0.025)$, and Sitta carolinensis Latham (White-breasted Nuthatch) $(\beta=0.98, P=0.003)$ (Table 3). In both the spring and autumn, Setophaga americana (L.) (Northern Parula) displayed significant associations with tree dominance ( $\beta=3.12$ [spring], $\beta=0.54$ [autumn], $P<$ $0.05)$ (Table 3). Poecile carolinensis (Audubon) (Carolina Chickadee) $(\beta=1.78$, $P<0.001)$ and Zonotrichia albicollis (Gmelin) (White-throated Sparrow) $(\beta=4.04$, $P=0.008$ ) had significant associations with DBH (Table 3).

\section{Apparent frequencies of occurrence}

Nested ANOVAs indicated that during spring, no bird models exhibited significant differences in apparent frequencies of occurrence in varying age categories. Analyses of autumn data indicated significant differences in 5 bird species (Fig. 4). Carolina Chickadees were more likely to occur in sites $15-23$ y of age $\left(F_{3,7}=2.77\right.$, $P=0.018$ ), Downy Woodpeckers were more likely to be encountered in mature bottomland-forest sites compared to restoration sites that were $7-14 \mathrm{y}$ old $\left(F_{3,7}=\right.$ 8.24, $P<0.001$ ), Colaptes auratus (L.) (Northern Flicker) were more likely to occur on mature bottomland-forest sites than on restoration sites between 1-14 y of age $\left(F_{3,7}=5.47, P<0.001\right)$, and both Red-headed Woodpeckers and Northern Parulas were more likely to occur in mature bottomland-forest reference sites than in restoration sites (Fig. 4).

\section{Discussion}

\section{Species diversity}

Nested ANOVAs resulted in no significant differences in diversity measurements at different age categories during spring and only significant differences with the youngest age category ( $<7$ years) in relation to all other categories during autumn. 
This result contrasted with our original hypothesis that sites of ages 15-23 y since restoration would have the highest diversity; however, we had a limited number of sites. Other studies have found diversity tends to increase with age of forest sites (Gram et al. 2003, Johnston and Odum 1956, Kricher 1973).

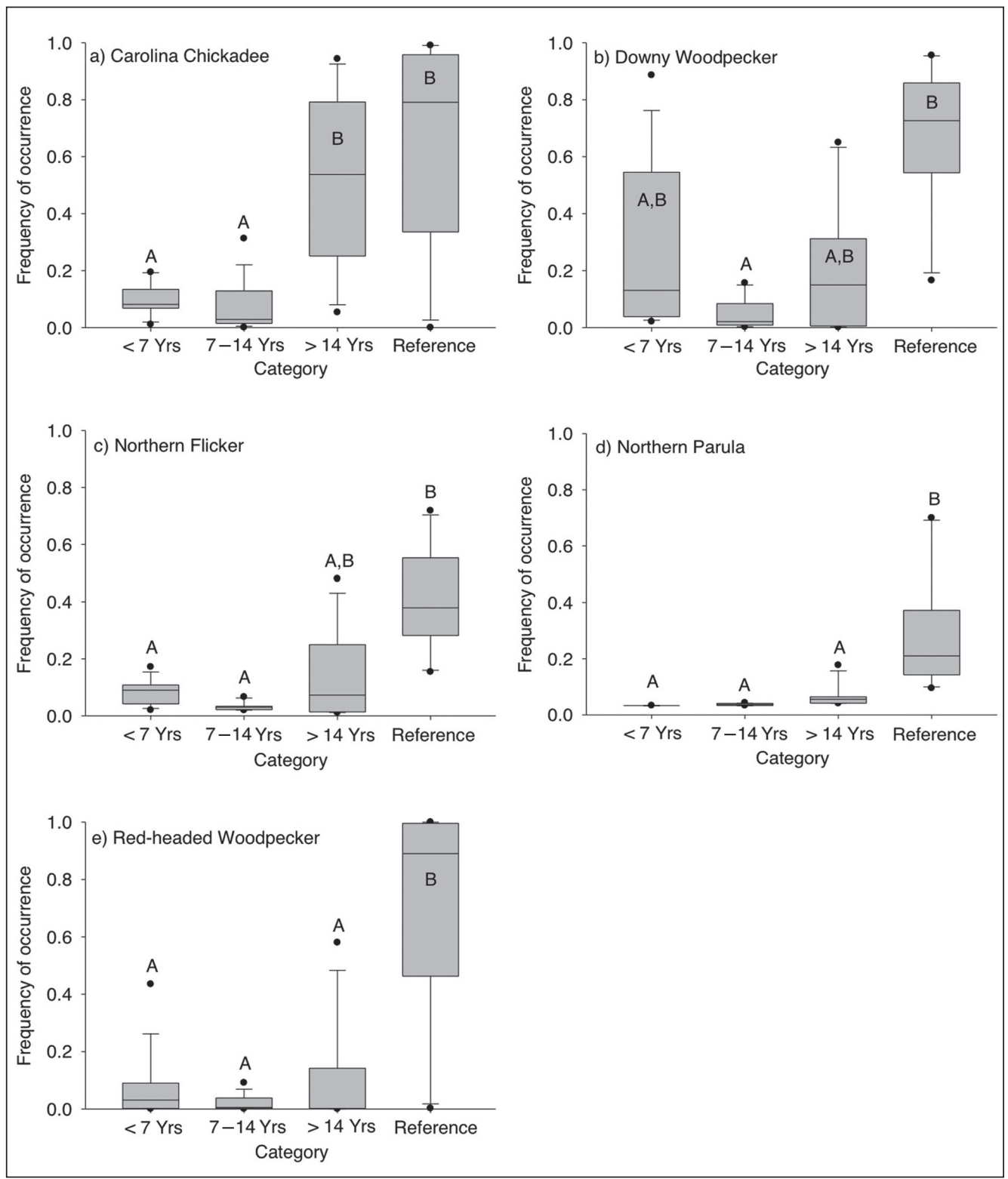

Figure 4. Apparent frequencies of occurrence for modeled species within different restoration-age categories. Median (bar), $10^{\text {th }}$ (lower error bar), $25^{\text {th }}$ (lower box), $75^{\text {th }}$ (upper box), and $90^{\text {th }}$ (upper error bar) percentiles are included. Outliers are represented with black dots. Points with the same letter represent no significant difference between means, which is based on Tukey-Kramer post hoc tests at $P=0.05$. 


\section{Habitat models}

Our predictive models for presence of Field Sparrow, Indigo Bunting, Northern Parula, and Red-eyed Vireo supported results reported in previous studies. In spring, The Field Sparrow's negative relationship with tree height, and in autumn, their positive association with distance to edge, and negative association with DBH was indicative of their preference for grasslands (Best 1977, Reidy et al. 2014). Previous literature showed that Indigo Buntings prefer areas with high herbaceous cover, which corresponds to the positive associations with groundcover and the curvilinear relationship to herbaceous height (Reidy et al. 2014, Stauffer and Best 1980). Northern Parula models also exhibited a significant positive association with tree dominance, which was supported by studies that indicated their preference for mature forests (Reidy et al. 2014, Rodewald and Brittingham 2007). Tree height was a significant positive indicator in the Red-eyed Vireo model. This finding was seen in previous studies that have noted that Red-eyed Vireos inhabit mature forests (James 1971, Reidy et al. 2014).

Some species, such as the American Goldfinch, Hermit Thrush, Summer Tanager, and Eastern Wood-Pewee, had models that were not supported by current literature. Our American Goldfinch model showed a significant positive association with distance to edge, but previous studies did not include this as an indicator variable. A number of other studies showed that American Goldfinches are primarily influenced by the presence of shrubs and the lack of saplings and mature trees (Mabry 2013, Stauffer and Best 1980). The Hermit Thrush model showed a significant positive association with $\mathrm{DBH}$, but a significant negative association with tree height. Previous observations have noted that Hermit Thrushes inhabit forested areas, which corresponded to the association with DBH but did not correspond with the tree-height relationship (Dellinger et al. 2012, Morse 1971). The Summer Tanager model included only shrub density as a positive significant indicator of presence. This result is in direct contrast with a previous study that showed that increasing shrub density had a negative effect on the presence of Summer Tanagers (Reidy et al. 2014). The Eastern Wood-Pewee model included a negative association with shrub height, which contrasted with a previous study that indicated a negative relationship with ground cover (Reidy et al. 2014).

We expected our data to be concordant with known habitat relationships, but had mixed results. There are several potential reasons on why some of the models described may not be supported by literature. For example, the autumn models included younger birds, which may prefer different habitat when compared to their adult counterparts. One example is post-fledging Wood Thrushes, which occupied early successional oak-Carya (hickory) forests rather than mature forests where adults were typically found (Anders et al. 1998). Some additional species, such as the Red-winged Blackbird, American Robin, Northern Cardinal, and White-throated Sparrow, had unusual indicators in their models but are considered to be generalist species (Blackwell and Dolbeer 2001, Dellinger et al. 2007, Kilgo et al. 1998, Rousseau et al. 2012, Whittaker and Marzluff 2009). Therefore, they are less responsive to habitat structure and do not necessarily produce predictive models indicative of their typical preference (Carrara et al. 2015, Hinsley et al. 2009, Julliard et al. 2006). 


\section{Apparent frequencies of occurrence}

Many of our models for bird species occurrence did not have significant differences in apparent frequency of occurrence when comparing restoration-age category, and only 5 species had any significant difference among age categories. Therefore, this finding may suggest that many of the species did not preferentially choose habitats during the surveying periods. Part of our sampling period included migration. During migration, habitat use tends to be more variable for bird species (Faaborg et al. 2010a, b; Petit 2000).

\section{Conclusions}

This study was one of the first times our restoration sites were surveyed to determine which bird species were utilizing these areas. Our results indicated that bottomland-hardwood forest-restoration sites provide habitat for a variety of birds from grassland specialists to forest specialists, with older sites having greater bird diversity. We detected several species of conservation concern during our surveys, such as Cerulean Warblers and Vireo bellii Audubon (Bell's Vireo) (Table 2). Our habitat models, while useful for exploration, did not result in discernible patterns or trends and therefore are of limited value for informing managers on management interventions they can use to increase and maintain bird populations at our sites. To better understand restoration efforts in the UMR, we recomend using our data as a baseline for future studies to determine if there are shifts in bird assemblages in these areas. We further recommend the inclusion of more-robust surveying techniques and the inclusion of more sites to provide data for better population estimates for surveyed bird species. Due to high likelihoods of flooding at our sites during spring, we also recommend that future research focuses on the breeding season and autumn migration.

\section{Acknowledgments}

We thank associate editor, Dr. Jeremy Kirchman, and 2 anonymous reviewers for their critical feedback and suggestions on our manuscript. We also thank Dr. Elizabeth Esselman, Dr. Jason Knouft, Dr. Laurel Hartley, Allison Pierce, Elizabeth Pansing, and Aaron Wagner for their comments and feedback on earlier iterations of the manuscript. Lastly, we thank Irene Weber, Benjamin Legal, Kathryn Leonard, Savannah Stabenow, Virginia Klein, Nicholas Horn, Charlie Deutsch, Ben McGuire, Lyle Guyon, and Brian Stoff for their assistance in traveling and field work. The project was supported with grants from the Southern Illinois University Edwardsville Graduate School, the Webster Groves Nature Study Society, Sigma $\mathrm{Xi}$, and the Illinois State Academy of Science.

\section{Literature Cited}

Akaike, H. 1974. A new look at the statistical model identification. IEEE Transactions on Automatic Control 19:716-723.

Anders, A.D., J. Faaborg, and F.R. Thompson. 1998. Postfledging dispersal, habitat use, and home-range size of juvenile Wood Thrushes. The Auk 115:349-358.

Au, L., D.E. Andersen, and M. Davis. 2008. Patterns in bird-community structure related to restoration of Minnesota dry oak savannas and across a prairie-to-oak woodland ecological gradient. Natural Areas Journal 28:330-341. 
Austin, M. 2007. Species-distribution models and ecological theory: A critical assessment and some possible new approaches. Ecological Modeling 200:1-19.

Best, L.B. 1977. Territory quality and mating success in the Field Sparrow (Spizella pusilla). The Condor 79:192-204.

Blackwell, B.F., and R.A. Dolbeer. 2001. Decline of the Red-Winged Blackbird population in Ohio correlated to changes in agriculture (1965-1996). The Journal of Wildlife Management 65:661-667.

Brawn, J.D. 2006. Effects of restoring oak savannas on bird communities and populations. Conservation Biology 20:460-469.

Carrara, E., V. Arroyo-Rodriguez, J.H. Vega-Rivera, J.E. Schondube, S.M. de Freitas, and L. Fahrig. 2015. Impact of landscape composition and configuration on forest specialist and generalist bird species in the fragmented Lacandona rainforest, Mexico. Biological Conservation 184:117-126.

Connell, J.H. 1978. Diversity in tropical rain forests and coral reefs. Science 199:1302-1310.

Davis, M.A., D.W. Peterson, P.B. Reich, M. Crozier, T. Query, E. Mitchell, J. Huntington, and P. Bazakas. 2000. Restoring savanna using fire: Impact on the breeding-bird community. Restoration Ecology 8:30-40.

Dellinger, R.L., P.B. Wood, P.D. Keyser, and G. Seidel. 2007. Habitat partitioning of 4 sympatric thrush species at 3 spatial scales on a managed forest in West Virginia. The Auk 124:1425-1438.

Dellinger, R.L., P.B. Wood, P.W. Jones, and T.M. Donovan. 2012. No. 261, In P.G. Rodewald (Ed.). Hermit Thrush (Catharus guttatus). Cornell Lab of Ornithology, Ithaca, NY. Available online at http://bna.birds.cornell.edu/bna/species/261. Accessed 21 February 2016.

Dey, D.C., W. Lovelace, J.M. Kabrick, and M.A. Gold. 2004. Production and early field performance of RPM seedlings in Missouri floodplains. Pp. 59-65, In C.H. Michler, P.M. Pijut, J. Van Sambeek, M. Coggeshall, J. Seifert, K. Woeste, R. Overton, F. Ponder Jr. (Eds.). Black Walnut in a new century, proceedings of the $6^{\text {th }}$ Walnut Council research symposium, 25-28 July, Lafayette, IN. General Technical Report NC-243. US Department of Agriculture, Forest Service, North Central Research Station, St. Paul, MN. 188 pp.

Environmental Systems Research Institute (ESRI). 2010. ArcGIS Desktop: Release 10. Redlands, CA.

Faaborg, J., R.T. Holmes, A.D. Anders, K.L. Bildstein, K.M. Dugger, S.A. Gauthreaux II, P.J. Heglund, K.A. Hobson, A.E. Jahn, D.H. Johnson, S.C. Latta, D.J. Levey, P.P. Marra, C. Merkord, E. Nol, S.I. Rothstein, T.W. Sherry, T.S. Sillett, F.R. Thompson III, and N. Warnock. 2010a. Conserving migratory land birds in the New World: Do we know enough? Ecological Applications 20:398-418.

Faaborg, J., R.T. Holmes, A.D. Anders, K.L. Blidstein, K.M. Dugger, S.A. Gauthreaux II, P.J. Heglund, K.A. Hobson, A.E. Jahn, D.H. Johnson, S.C. Latta, D.J. Levey, P.P. Marra, C. Merkord, E. Nol, S.I. Rothstein, T.W. Sherry, T.S. Sillett, F.R. Thompson, and N. Warnock. 2010b. Recent advances in understanding migration systems of New World land birds. Ecological Monographs 80:3-48.

Garvey, J., B. Ickes, and S. Zigler. 2010. Challenges in merging fisheries research and management: The Upper Mississippi River experience. Hydrobiologia 640:125-144.

Gram, W.K., P.A. Porneluzi, R.L. Clawson, J. Faaborg, and S.C. Richter. 2003. Effects of experimental forest management on density and nesting success of bird species in Missouri Ozark Forests. Conservation Biology 17:1324-1337. 
Hamel, P.B. 2003. Winter bird-community differences among methods of bottomland hardwood-forest restoration: Results after 7 growing seasons. Forestry 76:189-197.

Hanberry, B.B., J.M. Kabrick, H.S. He, and B.J. Palik. 2012. Historical trajectories and restoration strategies for the Mississippi River Alluvial Valley. Forest Ecology and Management 280:103-111.

Hinsley, S.A., R.A. Hill, P. Bellamy, R.K. Broughton, N.M. Harrison, J.A. Mackenzie, J.R. Speakman, and P.N. Ferns. 2009. Do highly modified landscapes favour generalists at the expense of specialists? An example using woodland birds. Landscape Research 34:509-526.

Hintze, J. 2007. NCSS 2007. NCSS, LLC. Kaysville, UT. Available online at www.ncss. com. Accessed 15 April 2014.

Hutto, R.L., S.M. Pletschet, and P. Hendricks. 1986. A fixed-radius point-count method for nonbreeding and breeding-season use. The Auk 103:593-602.

James, F.C. 1971. Ordinations of habitat relationships among breeding birds. The Wilson Bulletin 83:215-236.

Jensen, W.E. 2007. Missouri waterbirds and working lands: Technical report. Audubon Missouri:1-36.

Johnston, D.W., and E.P. Odum. 1956. Breeding bird populations in relation to plant succession of the Piedmont of Georgia. Ecology 37:50-62.

Julliard, R., J. Clavel, V. Devictor, F. Jiguet, and D. Couvet. 2006. Spatial segregation of specialists and generalists in bird communities. Ecology Letters 9:1237-1244.

Keating, K.A., and S. Cherry. 2004. Use and interpretation of logistic regression in habitatselection studies. Journal of Wildlife Management 68:774-789.

Kilgo, J.C., R.A. Sargent, B.R. Chapman, and K.V. Miller. 1998. Effects of stand width and adjacent habitat on breeding bird communities in bottomland hardwoods. The Journal of Wildlife Management 62:72-83.

King, S.L., and B.D. Keeland. 1999. Evaluation of the reforestation in the lower Mississippi River Alluvial Valley. Restoration Ecology 7:348-359.

King, S.L., D.J. Twedt, and R.R. Wilson. 2006. The role of the wetland reserve program in conservation efforts in the Mississippi River Alluvial Valley. Wildlife Society Bulletin 34:914-920.

Kirsch, E.M., P.J. Heglund, B.R. Gray, and P. McKann. 2013. Songbird use of floodplain and upland forests along the upper Mississippi River corridor during spring migration. The Condor 115:115-130.

Knutson, M.G., and E.E. Klaas. 1997. Declines in abundance and species richness of birds following a major flood on the upper Mississippi River. The Auk 114:367-380.

Knutson, M.G., J.P. Hoover, and E.E. Klaas. 1996. The importance of floodplain forests in the conservation and management of neotropical migratory birds in the Midwest. Pp. 1-21, In F.R. Thompson (Ed.). Management of Midwestern Landscapes for the Conservation of Neotropical Migratory Birds. General Technical Report NC-187. US Department of Agriculture, Forest Service, North Central Forest Experiment Station, St. Paul, MN. 208 pp.

Knutson, M.G., R.K. Hines, C.M. Sveum, T.J. Fox, and C.E. Korschgen. 1999. Floodplain forest songbirds of the Upper Mississippi River. The Passenger Pigeon 61:307-310.

Kricher, J.C. 1973. Summer bird-species diversity in relation to secondary succession on the New Jersey Piedmont. The American Midland Naturalist 89:121-137.

Mabry, C. 2013. Optimal shrub density for bird habitat in the midwestern United States. Ecological Restoration 31:63-68. 
Möckel, T., J. Dalmayne, B.C. Schmid, H.C. Prentice, and K. Hall. 2016. Airborne hyperspectral data predict fine-scale plant species diversity in grazed dry grasslands. Remote Sensing 8:133.

Morse, D.H. 1971. Effects of the arrival of the a new species upon habitat utilization by 2 forest thrushes in Maine. The Wilson Bulletin 83:57-65.

Nelson, E.C., and L.B. Wlosinski. 1999. Wetland birds of the Upper Mississippi River National Wildlife and Fish Refuge. The Passenger Pigeon 61:299-305.

Petit, D.R. 2000. Habitat use by landbirds along Nearctic-Neotropical migration routes: Implications for conservation of stopover habitats. Studies in Avian Biology 20:15-33.

Pizzio, R., C. Herrero-Jáuregui, M. Pizzio, and M. Oesterheld. 2016. Impact of stocking rate on species diversity and composition of a subtropical grassland in Argentina. Applied Vegetation Science 19:454-461.

Reidy, J.L., F.R. Thompson, and S.W. Kendrick. 2014. Breeding-bird response to habitat and landscape factors across a gradient of savanna, woodland, and forest in the Missouri Ozarks. Forest Ecology and Management 313:34-46.

Richter, L.A., R.L. Essner, and P.R. Minchin. 2010. A survey of bluff forest avifauna in southwestern Illinois. Transactions of the Illinois State Academy of Science 103:39-48.

Robinson, S.K., F.R. Thompson, T.M. Donovan, D.R. Whitehead, and J. Faaborg. 1995. Regional forest fragmentation and the nesting success of migratory birds. Science 267:1987-1990.

Rodewald, P.G., and M.C. Brittingham. 2007. Stopover habitat use by spring migrant landbirds: The roles of habitat structure, leaf development, and food availability. The Auk 124:1063-1074.

Romano, S.P. 2006. Tree composition and growth response to flooding in a hydrologically modified floodplain forest in southern Illinois, USA. Ph.D. Dissertation. Southern Illinois University-Carbondale, Carbondale, IL.

Romano, S.P. 2010. Our current understanding of the Upper Mississippi River system floodplain forest. Hydrobiologia 640:115-124.

Rousseau, P., A. Desrochers, and A.S. Hadley. 2012. Habitat selection and fidelity by White-throated Sparrows (Zonotrichia albicollis): Generalist species, specialist individuals? Canadian Journal of Zoology 90:595-601.

Roxburgh, S.H., K. Shea, and J.B. Wilson. 2004. The intermediate-disturbance hypothesis: Patch dynamics and mechanisms of species coexistence. Ecology 85:359-371.

Sauer, J.R., J.E. Hines, J.E. Fallon, K.L. Pardieck, D.J. Ziolkowski and W.A. Link. 2017. The North American Breeding Bird Survey: Results and analysis 1966-2015. Version 02.07.2017. Available online at https://www.mbr-pwrc.usgs.gov/bbs/. Accessed 8 October 2017.

Sparks, R.E. 2010. Forty years of science and management on the Upper Mississippi River: An analysis of the past and a view of the future. Hydrobiologia 640:3-15.

Stauffer, D.F., and L.B. Best. 1980. Habitat selection by birds of riparian communities: Evaluating effects of habitat alterations. The Journal of Wildlife Management 44:1-15.

Theiling, C.H., J.A. Janvrin, and J. Hendrickson. 2015. Upper Mississippi River restoration: Implementation, monitoring, and learning since 1986. Restoration Ecology 23:157-166.

Thompson, F.R., M.B. Robbins, and J.A. Fitzgerald. 2012. Landscape-level forest cover is a predictor of Cerulean Warbler abundance. The Wilson Journal of Ornithology 124:721-727.

Twedt, D.J., and C.R. Loesch. 1999. Forest area and distribution in the Mississippi alluvial valley: Implications for breeding bird conservation. Journal of Biogeography 26:1215-1224. 
Twedt, D.J., and J. Portwood. 1997. Bottomland hardwood reforestation for neotropical migratory birds: Are we missing the forest for the trees? Wildlife Society Bulletin 25:647-652.

Twedt, D.J., R.R. Wilson, J.L. Henne-Kerr, and D.A. Grosshuesch. 2002. Avian response to bottomland hardwood reforestation: The first 10 years. Restoration Ecology 10:645-655.

Twedt, D.J., P.B. Hamel, and M.S. Woodrey. 2008. Winter bird-population studies and Project Prairie Birds for surveying grassland birds. Southeastern Naturalist 7:11-18.

Van Der Maarel, E. 1975. The Braun-Blanquet approach in perspective. Vegetatio 30:213219.

Wells, J.V., D.K. Niven, and J. Cecil. 2005. The important bird areas program in the United States: Building a network of sites for conservation, state by state. PSW-GTR-191. US Department of Agriculture Forest Service, Southwest Research Station, Albany, CA. 5 pp.

Whittaker, K.A. and J.M. Marzluff. 2009. Species-specific survival and relative habitat use in an urban landscape during the postfledging period. The Auk 126:288-299.

Wilson, R.R., and D.J. Twedt. 2005. Bottomland hardwood establishment and avian colonization of reforested sites in the Mississippi Alluvial Valley. Pp. 341-352, In L.H. Fredrickson, S.L. King, R.M. Kaminski (Eds.). Ecology and management of bottomland hardwood systems: the state of our understanding. University of Missouri-Columbia, Gaylord Memorial Laboratory Special Publication 10. 431 pp. 